\title{
Emerging New Technology to Advance Personalized Medicine
}

\section{Toshihisa Ishikawa*}

RIKEN Omics Science Center, 1-7-22 Suehiro-cho, Tsurumi-ku, Yokohama 230-0045, Japan

Since initial sequencing and analysis of the human genome was accomplished [1,2], a huge effort has been put into medical research focused on associating genomic variations with individual phenotypes. Personalized medicine is often defined as "the right treatment for the right person at the right time." While the market for diagnostic tests and therapies that leverage this new science is growing, the biggest opportunities exist outside of the traditional healthcare sector. The Personalized Medicine market is projected to grow by 11.56 percent annually and is expected to reach US $\$ 148.4$ billion by 2015 . While personalized medicine is already being considered in drug development strategies, it is still at an early stage with respect to clinical applications that support patient-specific therapy.

Genetic polymorphisms and mutations in drug metabolizing enzymes, transporters, receptors, and other drug targets (e.g., toxicity targets) are linked to inter-individual differences in the efficacy and toxicity of medications as well as risk of genetic diseases. The interindividual variation in the rate of drug metabolism has been known for many years. Pharmacogenomics dealing with heredity and response to drugs is part of science that attempts to explain variability of drug responses and to search for the genetic basis of such variations or differences. Validation of clinically important genetic polymorphisms and development of new technologies to rapidly detect clinically important variants are critical issues for advancing personalized medicine.

The highest impact on personalized medicine is often seen for drugs with a narrow therapeutic index, with important examples emerging from treatment with antidepressants, oral anticoagulants, and chemotherapeutics, which are metabolized by CYP2D6/CYP2C9, $V K O R C 1$, and TPMT, respectively. To apply the ever increasing amounts of pharmacogenomics knowledge to clinical practice, specific dosage recommendations based on genotypes will have to be developed to guide the clinician; and these recommendations will have to be evaluated by the use of prospective clinical studies. Such efforts will lead to the development of personalized medicines, which would be expected to exhibit higher efficacy with fewer adverse drug reactions, thereby improving the therapeutic index for drugs whose pharmacokinetics, pharmacodynamics, and safety are influenced by pharmacogenetics. In fact, to improve drug safety, the FDA has started to update labels of previously approved drugs as new clinical and genetic evidence accrues [3]. Increases in efficacy and safety by the individualization of medical treatment may have benefits in financial terms, if information is presented to show that personalized medicine will be cost-effective in healthcare systems.

While the scientific community has largely accepted the utility of sequencing for research purposes [4,5], the use of the next-generation sequencing (NGS) technology in a clinical setting has yet to be fully addressed or accepted by the medical community. To effectively advance personalized medicine, it is necessary to be able to rapidly and conveniently test for patients' genetic polymorphisms and/or mutations. Recent years, new technologies are evolving to transform diagnostic devices for rapid testing at the Point-of-Care (POC). Portable devices are being engineered for use in a range of settings to perform robust assays for the diagnosis of disease that will improve patient management, and result in greater convenience and speed to answer. Current isothermal nucleic acid amplification methods include nucleic acid sequence-based amplification (NASBA) [6], transcriptionmediated amplification (TMA) [7], signal-mediated amplification of RNA technology (SMART) [8], helicase-dependent amplification (HDA) $[9,10]$, recombinase polymerase amplification (RPA) [11,12], loop-mediated amplification (LAMP) [13,14], cross-priming amplification (CPA) [15], smart amplification (SmartAmp) [16,17], rolling circle amplification (RCA) [18], and ramification amplification (RAM) [19]. Among them, SmartAmp has the capability of clinical genotyping [17].

The next important step is to incorporate pharmacogenomics data into routine clinical practice. A key requirement for the advancing personalized medicine resides in the ability of rapidly and conveniently testing patients' genetic polymorphisms and/or mutations. The POC diagnostics is a growing field that is gradually becoming more userfriendly with the introduction of portable devices and quicker nucleic acid detection. Successful POC diagnostics require 4 major elements, such as rapid reaction, low cost, low energy consumption, and simple analysis (with minimal technical training and inclusion of controls but no off-instrument processing or reagent preparation). Development of personalized medicine including POC diagnostics may require integration of various segments of biotechnology, clinical medicine, and pharmacology.

\section{References}

1. Lander ES, Linton LM, Birren B, Nusbaum C, Zody MC, et al. (2001) Initial sequencing and analysis of the human genome. Nature 409: 177-186.

2. Venter JC, Adams MD, Myers EW, Li PW, Mural RJ, et al. (2001) The sequence of the human genome. Science 291: 1304-1351.

3. Frueh FW, Amur S, Mummaneni P, Epstein RS, Aubert RE, et al. (2008) Pharmacogenomic biomarker information in drug labels approved by the United States food and drug administration: prevalence of related drug use. Pharmacotherapy 28: 992-998.

4. 1000 Genome Project Consortium (2010) A map of human genome variation from population-scale sequencing. Nature 467: 1061-1073.

*Corresponding author: Toshihisa Ishikawa, RIKEN Omics Science Center, 1-7-22 Suehiro-cho, Tsurumi-ku, Yokohama 230-0045, Japan, Tel: +81-503-9222; Fax: +81503-9216; E-mail: toshi-i@gsc.riken.jp

Received January 22, 2012; Accepted January 23, 2012; Published January 26 2012

Citation: Ishikawa T (2012) Emerging New Technology to Advance Personalized Medicine. J Pharmacogenom Pharmacoproteomics 3:e113. doi:10.4172/21530645.1000e113

Copyright: (c) 2012 Ishikawa T. This is an open-access article distributed under the terms of the Creative Commons Attribution License, which permits unrestricted use, distribution, and reproduction in any medium, provided the original author and source are credited. 
Citation: Ishikawa T (2012) Emerging New Technology to Advance Personalized Medicine. J Pharmacogenom Pharmacoproteomics 3:e113. doi:10.4172/2153-0645.1000e113

Page 2 of 2

5. Rubenstein K (2010) Next-generation sequencing technologies: applications and markets. Insight Pharma Reports. Cambridge Healthcare Institute publication, Needham, MA, USA.

6. Gracias KS, McKillip JL (2007) Nucleic acid sequence-based amplification (NASBA) in molecular bacteriology: a procedural guide. J Rapid Methods Autom Microbiol 15: 295-309.

7. Hofmann WP, Dries V, Herrmann E, Gartner B, Zeuzem S, et al. (2005) Comparison of transcription mediated amplification (TMA) and reverse transcription polymerase chain reaction (RT-PCR) for detection of hepatitis C virus RNA in liver tissue. J Clin Virol 32: 289-293.

8. Wharam SD, Hall MJ, Wilson WH (2007) Detection of virus mRNA within infected host cells using an isothermal nucleic acid amplification assay: marine cyanophage gene expression within Synechococcus sp. Viol J 4: 52

9. Jeong YJ, Park K, Kim DE (2009) Isothermal DNA amplification in vitro: the helicase-dependent amplification system. Cell Mol Life Sci 66: 3325-3336.

10. Vincent M, Xu Y, Kong H (2004) Helicase-dependent isothermal DNA amplication. EMBO Rep 5: 795-800.

11. Lutz S, Weber P, Focke M, Faltin B, Hoffman J, et al. (2010) Microfluidic lab-ona-foil for nucleic acid analysis based on isothermal recombinase polymerase amplification (RPA). Lab Chip 10: 887-893.

12. Piepenburg O, Williams CH, Stemple DL, Armes NA (2006) DNA detection using recombination proteins. PLoS Biol 4: e204

13. Curtis KA, Rudolph DL, Owen SM (2009) Sequence-specific detection method for reverse transcription, loop-mediated isothermal amplification of HIV-1. J Med Virol 81: 966-972.

14. Mori Y, Nakatomi T (2009) Loop-mediated isothermal amplification (LAMP): a rapid, accurate, and cost-effective diagnostic method for infectious diseases. J Infect Chemother 15: 62-69.

15. Fang R, Li X, Hu L, You Q, Li J, et al. (2009) Cross-priming amplification for rapid detection of Mycobacterium tuberculosis in sputum specimens. $\mathrm{J}$ Clin Microbiol 47: 845-847.

16. Mitani Y, Lezhava A, Sakurai A, Horikawa A, Nagakura M, et al. (2009) Rapid and cost-effective SNP detection method: application of SmartAmp2 to pharmacogenomics research. Pharmacogenomics 10: 1187-1197.

17. Ishikawa T, Hayashizaki Y (2012) Clinical SNP detection by the SmartAmp method. Methods Mol Biol.

18. Johne R, Müller H, Rector A, van Ranst M, Stevens H (2009) Rolling-circle amplification of viral DNA genomes using phi29 polymerase. Trends Microbio 17: $205-211$.

19. Yao B, Huang H, Sun C, Wang Z, Fan Y, et al. (2009) Quantitative analysis of zeptomole microRNAs based on isothermal ramification amplification. RNA 15: $1787-1794$ 